\title{
Multiple-true-false questions reveal more thoroughly the complexity of student thinking than multiple-choice questions: a Bayesian item response model comparison
}

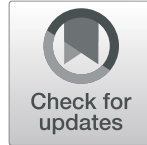

\author{
Chad E. Brassil and Brian A. Couch * (D)
}

\begin{abstract}
Background: Within undergraduate science courses, instructors often assess student thinking using closed-ended question formats, such as multiple-choice (MC) and multiple-true-false (MTF), where students provide answers with respect to predetermined response options. While MC and MTF questions both consist of a question stem followed by a series of options, MC questions require students to select just one answer, whereas MTF questions enable students to evaluate each option as either true or false. We employed an experimental design in which identical questions were posed to students in either format and used Bayesian item response modeling to understand how responses in each format compared to inferred student thinking regarding the different options.
\end{abstract}

Results: Our data support a quantitative model in which students approach each question with varying degrees of comprehension, which we label as mastery, partial mastery, and informed reasoning, rather than uniform random guessing. MTF responses more closely estimate the proportion of students inferred to have complete mastery of all the answer options as well as more accurately identify students holding misconceptions. The depth of instructional information elicited by MTF questions is demonstrated by the ability of MTF results to predict the MC results, but not vice-versa. We further discuss how MTF responses can be processed and interpreted by instructors.

Conclusions: This research supports the hypothesis that students approach MC and MTF questions with varying levels of understanding and demonstrates that the MTF format has a greater capacity to characterize student thinking regarding the various response options.

Keywords: Assessment, Bayesian analysis, Item response theory, Mixed conceptions, Multiple-choice, Multiple-truefalse, Question format, Undergraduate

\section{Introduction}

While assessment instruments have been used commonly in undergraduate science education to measure student achievement for the purposes of assigning grades, these instruments can also play a formative role in the learning process (Angelo, 1998; Handelsman, Miller, \& Pfund, 2007). In particular, formative assessment provides an opportunity for students to practice answering questions, gives instructors information they can use to alter their teaching, and creates opportunities

\footnotetext{
* Correspondence: bcouch2@unl.edu

School of Biological Sciences, University of Nebraska, 204 Manter, Lincoln, NE 68588-0118, USA
}

for students to receive feedback on their progress (Black \& Wiliam, 2009; Ericsson, Krampe, \& Tesch-romer, 1993). Given that instructors often infer student understanding based on aggregate responses to individual questions, assessment items must provide accurate information that can be used to diagnose and give feedback to students with respect to specific conceptions.

When designing an instrument or activity, assessment developers choose a question format (i.e., item type) that meets their needs while considering various practical constraints, such as limited assessment time and grading resources. In many cases, instructors use closed-ended formats where students select from a predetermined list 
of response options. Multiple-choice (MC) questions represent a common closed-ended format used throughout undergraduate science education (Eagan et al., 2014). By having students select a single correct answer among a list of plausible distractors, MC questions provide an estimate of how many students endorse correct versus incorrect ideas. The $\mathrm{MC}$ format has been employed in a wide variety of contexts, from low-stakes daily activities to high-stakes assessments determining professional advancement. Education researchers have also developed tools that employ MC questions for formative purposes. For example, MC "clicker" questions serve as in-class check-ins that can facilitate student discussion and provide real-time feedback to inform instructional decisions (Crouch \& Mazur, 2001; Hubbard \& Couch, 2018; Vickrey, Rosploch, Rahmanian, Pilarz, \& Stains, 2015; Wood, 2004). Similarly, numerous MC research-based assessments (RBAs) and concept inventories have been developed to help instructors diagnose student understandings and guide larger-scale changes to curriculum and instruction (e.g., Hestenes, Wells, \& Swackhamer, 1992; Smith, Wood, \& Knight, 2008).

Despite the widespread use of the MC format, research into student mental models has exposed potential limitations of MC questions. Students often have mixed and incoherent understandings of natural phenomena, meaning that they can simultaneously hold both correct and incorrect ideas regarding particular concepts (Nehm \& Reilly, 2007; Nehm \& Schonfeld, 2008). This presents a problem for the MC format because students who select the correct answer may still consider one or more of the distractors to also be correct (Parker et al., 2012). In light of this issue, some assessment developers have adopted multiple-response formats that allow students to evaluate each response option (Kalas, O'Neill, Pollock, \& Birol, 2013; Newman, Snyder, Fisk, \& Wright, 2016).

Multiple-true-false (MTF) questions represent a multiple-response format that retains the question stem and response option structure of MC questions but requires students to separately mark each option as true or false, rather than selecting one correct option. Early work in comparing MC and MTF questions focused on quantifying reliability, difficulty, and the amount of time needed to complete questions (Dudley, 2006; Frisbie, 1992; Haladyna, Downing, \& Rodriguez, 2002; Javid, 2014). In general, the MTF format yields superior internal test reliability determined by coefficient alpha, even when accounting for item number and test duration (Couch, Hubbard, \& Brassil, 2018; Frisbie \& Sweeney, 1982; Kreiter \& Frisbie, 1989). While students are known to employ test-taking strategies (e.g., option length comparisons, option elimination) for MC questions (Ellis \& Ryan, 2003; Kim (Yoon \& Goetz, 1993; Stenlund, Eklöf, \& Lyrén, 2017), less is known about how students approach MTF questions. Studies produce conflicting results with respect to how response patterns differ based on being asked to only endorse true statements versus being asked to also mark false statements (Cronbach, 1941; Pomplun \& Omar, 1997). Recent work has highlighted how MTF questions reveal the prevalence of mixed and partial understandings among students, even within upper-division courses consisting mostly of graduating seniors (Couch, Wood, \& Knight, 2015; Parker et al., 2012).

To understand the effects of question format on student responses, members of our research team previously conducted a within-subjects controlled experiment where similar groups of students answered identical questions in either the MC or MTF format (Couch et al., 2018). On average, nearly half of the students who correctly answered a MC question likely would have endorsed one or more of the remaining distractors, if given the opportunity. The MC format underestimated the proportion of students who would have endorsed the correct answer as well as each incorrect distractor in the MTF format. These findings collectively suggested that the MC format systematically misrepresents student thinking by failing to reflect the partial understandings that most students hold regarding the various response options. This has implications when using the MC format for formative and diagnostic purposes because it could lead instructors and students to make instructional decisions based on incomplete or inaccurate information.

While initial analyses suggested that MTF questions reveal partial understandings otherwise obscured by the MC format, these results warranted further investigation to understand how students process the two question formats and address alternative explanations for previous results. A major difference between the MC and MTF formats lies in their putative guess rates. When students engage in uniform random guessing, each MC option will be selected roughly $25 \%$ of the time, whereas each MTF statement will be endorsed $50 \%$ of the time. Thus, the previous finding that students endorse both correct and incorrect statements more in the MTF format than the $\mathrm{MC}$ format could be partially explained by the different guess rates. Further, the MTF format could have induced acquiescence bias, where students systematically tend to mark true for MTF statements, leading to inflated endorsement rates based on non-content reasons (Cronbach, 1941). Finally, the presence of weak distractors could have led the MC format to overestimate question mastery because adequate alternatives were not presented to attract students with partial understandings.

Response modeling represents an approach researchers can use to estimate underlying parameters that are not directly apparent in raw responses. Item 
response theory (IRT) models person ability and item parameters (i.e., difficulty, discrimination, and pseudoguessing) based on student responses across an instrument (de Ayala, 2008). When applied to MC questions, IRT typically considers responses as dichotomous correct/incorrect variables, and thus, does not provide insights on student thinking regarding the various response options. However, polytomous responses from MC option selections can be incorporated into IRT models, known as nominal response models, enabling one to estimate the probability of a student selecting a particular response option based on a student's overall ability level (Bock, 1972). This approach can lead to more accurate person ability estimates (Baker \& Kim, 2004; Briggs, Alonzo, Schwab, \& Wilson, 2006) and more refined information regarding the relative attractiveness of the different response options for students at different ability levels (Bolt, Cohen, \& Wollack, 2001; Thissen, Steinberg, \& Fitzpatrick, 1989). However, since the $\mathrm{MC}$ format intrinsically depends on students selecting only one answer, this approach requires modification for use with MTF questions, where students separately evaluate each response option associated with a question stem.

We sought to develop a response model that could simultaneously be applied to both MC and MTF questions to understand how student thinking becomes manifest in responses to each format. We developed a variant of a Bayesian item response model that included MC and MTF responses, incorporating information on how students responded to each option. In part, our model represents a reparameterization of a traditional IRT model. Terms such as item difficulty and discrimination map onto particular IRT parameters and also frame an implied interpretation of student cognitive processing. We utilize the unique nature of our paired, experimental questions to develop a model that can be derived from IRT parameters, such as item difficulty, but that ultimately parameterizes new terms. These new terms frame the interpretation of our analysis in terms of student understanding with respect to different response options. Consequently, these terms imply a different cognitive model of student processing that can guide instructors in considering specific student misconceptions.

In developing this model, we wanted to understand how student responses varied with respect to specific questions, rather than focusing on how well each format estimates overall student ability levels. Specifically, we aimed to address several related research questions.

- How well do MC and MTF response rates approximate the proportion of students that have complete, partial, or little understanding of the response options?
- To what extent do MC and MTF responses reflect random guessing?

- To what extent can responses for each format be used to predict responses to the other format?

- What is the potential utility of $\mathrm{MC}$, as compared to MTF, to reveal student question mastery, even with different distractor characteristics?

- How should MTF responses be processed and interpreted by educators in light of the model?

In answering these questions, we sought to generate practical insights that instructors can follow to use closed-ended assessments in a manner that best captures the complexity of student thinking.

\section{Methods}

\section{Experimental design}

The current analyses utilize a previously reported dataset (Couch et al., 2018) but do not overlap with this previous publication. Briefly, we implemented a crossover experimental design within four unit exams of an introductory biology course. Our question development aligned with typical instructor construction and interpretation of unit exams, where questions do not undergo "think-aloud" interviews and individual questions are used to make conclusions about student understanding of specific topics. Experimental MC $\times$ MTF questions were written that contained one correct/true option and three incorrect/false distractors, enabling them to be presented with identical wording in either the MC or MTF formats (i.e., the formats differed only in how students input their respective answers). We followed established item writing guidelines to maximize the extent to which questions were clear, concise, and well-targeted for the intended group (Frey, Petersen, Edwards, Pedrotti, \& Peyton, 2005). For each experimental question, the MC form of the question was given on one exam version, and the MTF form was given on the other exam version. A total of $36 \mathrm{MC} \times \mathrm{MTF}$ questions were implemented across the semester in a counterbalanced fashion, such that each student answered half the experimental questions in the MC form and half in the MTF form. This design made our investigation of question format robust to issues of question writing because the same wording appeared across both formats. These experimental questions were embedded in an exam with a mix of additional MC and MTF questions. All MC and MTF questions had four response options or statements, and the entire MTF section on each exam had a relatively even balance of questions with one, two, or three true statements. Alternate exam versions were distributed to students in a semi-random fashion, and students recorded their responses to closed-ended questions on Scantron sheets. 
For an individual $\mathrm{MC} \times \mathrm{MTF}$ question, the $\mathrm{MC}$ response options and corresponding MTF statements appeared in an identical order on exams. Across the different MC $\times$ MTF questions, the position of the correct/ true option varied in a semi-random fashion, such that each of the four answer positions received roughly equal representation. For the purpose of data analysis and presentation, the response options have been reordered: the first option corresponds to the correct/true option and the remaining incorrect/false options are ordered sequentially according to their average selection/endorsement rates, with the most frequently selected distractor coming first and the least frequently selected distractor coming last. Thus, the correct response to $\mathrm{MC}$ questions will be $\mathrm{A}$, and options $\mathrm{B}-\mathrm{D}$ will represent the distractors in order of decreasing selection/endorsement rates. MTF question responses will be represented by a four-digit code, corresponding to answers to each of the four statements. For example, a fully correct answer will be represented by TFFF, meaning that the student correctly answered true for the true statement and correctly answered false for the three false statements.

A total of 194 students consented to have their exam data released for research purposes, representing $78 \%$ of total course enrollment. Student data was included in the data set on an available case basis (i.e., students who missed one or more exams typically due to course withdrawal still had their available data included). All student data was analyzed at once after the semester; we did not separately analyze the data for each exam. Students had a raw average of $72 \pm 13 \%$ SD across the four exams, including the other non-experimental questions. Additional file 1: Supplementary Material 1 provides a summary of student demographic characteristics. This research was classified as exempt from the Institutional Review Board review, Project ID 14314.

\section{Analyses}

A Bayesian approach to modeling student responses (Fox, 2010) allowed us to construct a model of student thinking and utilize that model to compare the two question formats. Our response model builds on the idea that students have certain incoming understandings of the various response options when they encounter an experimental exam question. These understandings are then translated into specific responses depending on the format of the question. In the process of model construction, we formulated latent (i.e., not directly observed) parameters describing student approaches, and we calculated a set of shared parameters for each $\mathrm{MC} \times$ MTF question to simultaneously predict aggregate population-level student response probabilities in both formats. At the same time, we accounted for differences in individual student performance, a hierarchical feature of the data for which a Bayesian approach is well suited when used in combination with a structured model. In our case, the utility of priors was not a motivating feature of a Bayesian approach. Therefore, weak, or vague, priors were utilized throughout, essential in eliminating the influence of priors in this analysis. The repeated measures aspect of the data in which each student answers multiple questions was accounted for when we included a student ability parameter (see individual student performance, as described below). We fit the best parameters for alternative models and compared the fit of each model to the empirical data. In the best-fit model, students process questions via a decision tree involving mastery, partial mastery, informed reasoning, and endorsement bias (Fig. 1). Additional file 1: Supplementary Material 2 includes a mathematical description of the Bayesian model for the most supported model.

\section{Mastery}

The mastery component modeled the proportion of students that had correct understandings of all the response options and therefore provided a fully correct response in either format (i.e., they answered A for the MC format or TFFF for the MTF format). While all students with mastery answered A or TFFF, not all students with these responses fell under mastery because some students may have arrived at fully correct answers via another approach (i.e., partial mastery or informed reasoning, as described below). Mastery was a latent variable inferred based on student responses (i.e., it was not determined using an external tool) and limited to the context of the particular statement options in each question. While this definition of mastery has limitations for extrapolation beyond the statement options, it is appropriately used as a comparison metric between the two formats within this study.

\section{Partial mastery}

Partial mastery modeled the proportion of students that had a correct understanding of the correct/true statement but also mistakenly believed that one of the distractors was also true, albeit to a lesser extent. For a MC question, these students answered A, and for an MTF question, they endorsed the correct answer along with the first distractor (i.e., they answered TTFF). In the first alternative structure, students with partial mastery endorsed the correct answer in the MTF format along with either the first or second distractor (i.e., they answered TTFF or TFTF). In the second alternative structure, students with partial mastery endorsed the correct answer in the MTF format along with either the first, second, or third distractor (i.e., they answered TTFF, TFTF, or TFFT). Again, while all students with partial mastery 


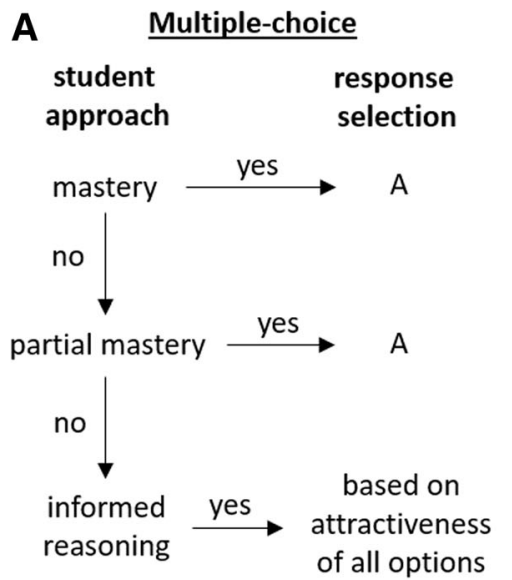

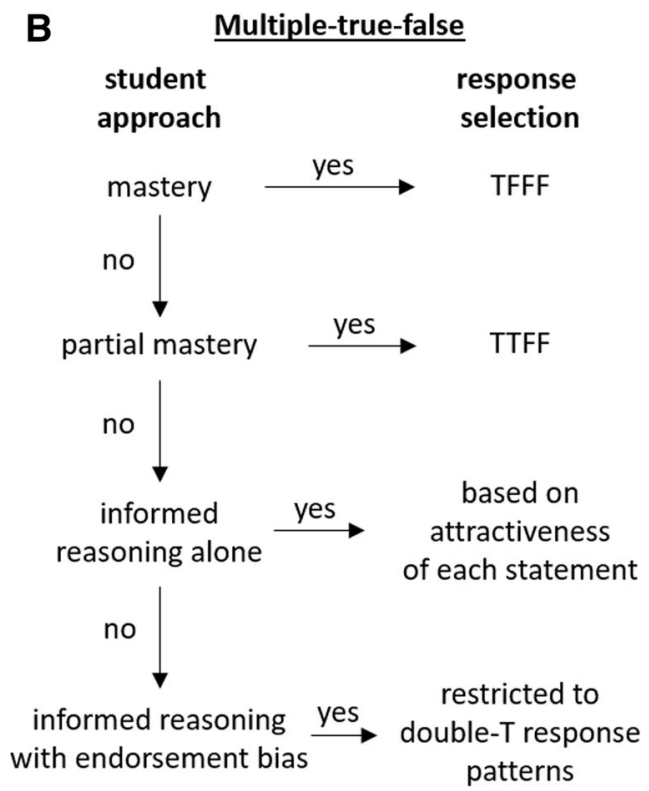

Fig. 1 Decision tree representation of the best-fit model describing how students approached a multiple-choice (MC) and $\mathbf{b}$ multiple-true-false (MTF) questions. In the MC format, "A" represented selection of the correct statement. Students with mastery or partial mastery were modeled as selecting $A$ in the MC format. Students outside these categories engaged in informed reasoning based on the attractiveness values of all the options. In the MTF format, "T" and "F" represent true and false answer selections for a question, with the first position referring to the answer to the true statement and the remaining positions referring to answers to the false statements in order of decreasing attractiveness. Students with mastery provided a fully correct answer of TFFF in the MTF format. Students with partial mastery answered TTFF, meaning they correctly said true for the true statement, incorrectly said true for the most attractive false statement, and correctly said false for the remaining false statements. Students outside the mastery and partial mastery categories engaged in informed reasoning alone based on the attractiveness of each statement. An additional group of students engaged in informed reasoning with endorsement bias, which restricted their responses to patterns with two true answers

answered A or TTFF, not all students with these responses fell under partial mastery because they may have arrived at these answers via another approach (i.e., informed reasoning, as described below).

\section{Informed reasoning based on option attractiveness}

Students that did not have either mastery or partial mastery were modeled as using informed reasoning to choose a response based on the underlying attractiveness of the various response options/statements. Attractiveness values, latent variables in the model, were estimated for each option/statement, representing the independent probability of endorsing each option/statement. On a pedagogical level, attractiveness values provide insights into the degree to which struggling students were inclined to select/endorse the various options and, therefore, the degree to which students consider distractors as valid concepts.

In the MTF format, the probability that a student using informed reasoning would endorse a statement as being true was calculated as the attractiveness of that option. Thus, the probability of selecting a particular answer pattern for a MTF question was the product of the associated attractiveness values for the four individual statements. For the MC format, the probability that a student using informed reasoning would select a particular option was based on the probabilities of the four different MTF answer patterns specifying singular endorsement of each option (i.e., TFFF, FTFF, FFTF, and FFFT corresponding to $\mathrm{A}, \mathrm{B}, \mathrm{C}$, and $\mathrm{D}$, respectively). The probability of selecting a particular MC option was calculated as the probability of that particular response pattern divided by the sum of all four single- $T$ probabilities (see Additional file 1: Supplementary Material 3 for further description).

\section{Informed reasoning with MTF endorsement bias}

Within the MTF format, we also recognized that students may have tendencies to endorse certain answer patterns instead of considering each statement independently from other statements. Students operating under double- $\mathrm{T}$ endorsement bias still weighed the various statements according to their underlying attractiveness values but had a bias to select among the answer patterns containing two true answers (i.e., TTFF, TFTF, TFFT, FTTF, FTFT, or FFTT) above that expected based on attractiveness alone. In the first alternative structure, students operating under multi-T bias favored response 
patterns containing two or more true answers. In the second alternative structure, rather than calculating double-T bias for each question, a double-T bias value was calculated for each student based on the hypothesis that each student may have their own intrinsic tendency for this bias.

Note, the model structure for endorsement bias was superficially similar to partial mastery in that students were restricted to a subset of all the possible answer permutations. However, endorsement bias applies to those students engaged in informed reasoning with the correct answer being weighed against other options. In contrast, partial mastery describes students with not only a defined understanding of the correct answer, but also a defined misunderstanding of a distractor.

\section{Random guessing}

We tested an additional component to account for random guessing. Students engaged in random guessing were modeled as having a $25 \%$ probability of selecting each MC option and a 50\% chance of selecting true for each MTF statement (i.e., uniform random). In one case, random guessing was an alternative to informed reasoning. In another case, random guessing was modeled in addition to informed reasoning (i.e., students not in mastery, partial mastery, or informed reasoning were modeled to be engaged in random guessing).

\section{Individual student performance}

We included an additional individual student performance parameter to account for differences in the particular sample of students taking each question version. Each individual student performance parameter was based on the extent to which they demonstrated mastery across all the questions. The probability of a student answering a particular question via mastery was thus modeled based on the mastery level of the question as well as the student's individual performance across all questions.

\section{Relationship to IRT models}

We developed a specifically structured polytomous item response model. In terms of a traditional binary item response model, our model maps onto the equivalent of a one-parameter logistic model, or Rasch model (Rasch, 1960). The item difficulty is the negative of the question mastery parameter $\left(-u_{i}\right)$, and we model the ability level for each individual $\left(v_{j}\right)$. We did not include the discrimination parameter from a two-parameter logistic model because our interest is not in determining an individual, assessment-level ability. Rather, our interest is in contrasting these two question formats in their ability to provide information on the item difficulty and on the attractiveness of options for individual questions. We included a structure accounting for informed reasoning, which is similar to but provides richer information than the pseudo-guessing parameter of the three-parameter logistic model. We model a complex polytomous response and construct a unique model relating $\mathrm{MC}$ responses to MTF responses with a common set of parameters. Therefore, our model is a derived version of IRT, specifically structured for our comparison between these question formats. Our data consists of the joint responses across all students and across both formats of the question.

\section{Model fit}

The above structures were each modeled at the question level. The independent variables were the question format being answered and the student answering. The response variable in all models was the selection of a MC option or selection of true or false for the four MTF statements for each question by each student. All parameters with the exception of question-level mastery were modeled hierarchically (i.e., by fitting mean and dispersion parameters for the entire question distribution from which individual question values were drawn). In mixed-models parlance, this equated to considering these parameters as "random effects." Question-level mastery was fit independently for each question (i.e., as a "fixed effect"). This decision was informed by preliminary analyses indicating that mastery levels were fairly uniformly spread, while the values of other question-level parameters clustered into distributions (Additional file 1: Supplementary Material 4).

To justify the inclusion of each structure, model comparisons were made using a Watanabe-Akaike information criterion (WAIC), a recently recommended Bayesian analog to AIC (Gelman, Hwang, \& Vehtari, 2014; Vehtari, Gelman, \& Gabry, 2017). The best-fit model was compared with and without each parameter structure, resulting in nested models. Components were retained when their absence increased WAIC by more than 2, a suggested threshold for model comparison (Gelman et al., 2014). For nested models, a decrease in WAIC of less than 2 was assumed to be consistent with a component of little to no real effect and therefore dropped for parsimony. Models were also compared with non-nested, alternative structures, and the structure that provided the largest WAIC decrease was selected for model inclusion. To be clear, the structure of the most supported model is derived from the data and not a single a priori hypothesis. However, the structures are based on potential types of student processing, and our analysis is consistent with multi-model inference (Burnham \& Anderson, 2002).

Overall, priors were non-informative or weak-informative so as to have no practical influence on the model 
fit. Non-informative, uniform priors were used for all parameters with beta distributions $(\alpha=1$ and $\beta=1$ for those having probabilities constrained between 0 and 1). Half-Cauchy priors (Gelman, 2006) were used as weak-information priors $(\sigma=2.5)$ for the dispersal parameter of the hierarchical probability values (i.e., the distributions from which the question-level values were drawn). Weak-information normal priors (centered at the mean with a standard deviation $=2$ ) were used for mastery parameters, which were themselves components in a logit link function (Additional file 1: Supplementary Material 2). Model parameterization was conducted using the program Stan in $\mathrm{R}$ with the package rstan (Stan Development Team, 2017). Model comparison via WAIC was calculated in $\mathrm{R}$ using the package loo.

\section{Results}

\section{Model identification}

By comparing the fit statistics for various model permutations, we arrived at a best-fit model that included structures for mastery, partial mastery, informed reasoning based on option attractiveness, informed reasoning with endorsement bias, and individual student performance (Table 1, model A). Comparisons among alternative structures enabled us to understand student response tendencies. The most supported model provides a hierarchical and probabilistic representation of how students processed MC and MTF questions (Fig. 1). The parameter distributions for the various parameters were consistent with either the hierarchical or independent nature of each component (Additional file 1: Supplementary Material 4). The best-fit model also produced reasonable posterior predictive checks in which computed values were compared to observed response data.

With respect to alternative model structures, students with partial mastery were best modeled as selecting TTFF (Table 1, model A), rather than alternative models in which these students correctly selected true for the true statement and also selected true for any one of the two most attractive false statements (model D) or any one of the three false statements (model E). These findings support the notion that some students had an understanding of three out of four response options, but they held a misconception regarding the most attractive distractor. For most questions, partial mastery made little contribution, but for roughly seven questions, partial mastery enabled a sufficiently better fit to warrant retention in the final model (Additional file 1: Supplementary Material 4).

Students without mastery or partial mastery were best modeled as engaging in informed reasoning based on statement attractiveness. This structure was the most strongly supported of any element in the model in that replacing it with random guessing resulted in the largest WAIC increase (model F). While some students within this group selected answers based solely on their attractiveness, we also tested structures in which students engaged in informed reasoning biased their responses toward patterns with two true answers (double-T endorsement bias) or multiple true answers (multi-T

Table 1 Support for model structures. Model A was the best-supported model in italics. Changes in structures from model A are indicated. The differences in WAIC compared to model A were greater than 13 for all models, except model J. Given that model J was more complex, that model A was nested within model J, and that the WAIC difference was small (less than 2), the simpler model A was accepted for parsimony

\begin{tabular}{|c|c|c|c|c|c|}
\hline & Model structures & WAIC & $\triangle$ WAIC & WAIC SE & $P_{\text {WAIC }}$ \\
\hline $\bar{A}$ & $\begin{array}{l}\text { Mastery, TTFF partial mastery, informed reasoning based on attractiveness, } \\
\text { informed reasoning with double-T endorsement bias, individual student performance }\end{array}$ & $18,520.7$ & 0 & 200.0 & 342.1 \\
\hline B & - remove question-level mastery & $18,927.0$ & -406.3 & 199.1 & 288.7 \\
\hline C & - remove TTFF partial mastery & $18,566.0$ & -45.3 & 200.2 & 328.9 \\
\hline $\mathrm{D}$ & $\begin{array}{l}\text { - remove TTFF partial mastery } \\
\text { + replace with TTFF-TFTF partial mastery }\end{array}$ & $18,534.3$ & -13.6 & 199.7 & 341.8 \\
\hline $\mathrm{E}$ & $\begin{array}{l}\text { - remove TTFF partial mastery } \\
\text { + replace with TTFF-TFTF-TFFT partial mastery }\end{array}$ & $18,536.5$ & -15.8 & 199.5 & 333.5 \\
\hline $\mathrm{F}$ & $\begin{array}{l}\text { - remove informed reasoning based on attractiveness } \\
\text { + replace with random guessing }\end{array}$ & $19,582.4$ & -1061.7 & 203.4 & 268.4 \\
\hline G & - remove double-T bias & $18,656.1$ & -135.4 & 201.4 & 330.3 \\
\hline $\mathrm{H}$ & $\begin{array}{l}\text { - remove double-T bias } \\
\text { + replace with multi-T bias }\end{array}$ & $18,533.4$ & -12.7 & 200.3 & 344.6 \\
\hline । & $\begin{array}{l}\text { - remove question-level double-T bias } \\
\text { + replace with global double-T bias for each student }\end{array}$ & $18,539.6$ & -18.9 & 200.0 & 325.1 \\
\hline J & $\begin{array}{l}\text { + add random guessing for students not in mastery, partial mastery, or } \\
\text { informed reasoning }\end{array}$ & $18,519.9$ & +0.8 & 199.9 & 356.1 \\
\hline K & - remove individual student performance & $19,448.5$ & -927.8 & 196.7 & 180.2 \\
\hline
\end{tabular}


endorsement bias). We found that double- $\mathrm{T}$ bias was more strongly supported than multi-T bias (model $\mathrm{H}$ ). While the distribution of double- $\mathrm{T}$ bias values was closely clustered, there was stronger support for question-level bias values over student-level bias (model I). To further address the possibility of random guessing, we tested an additional model in which students without mastery, partial mastery, or informed reasoning engaged in random guessing (model J). For a nested model, WAIC did not decrease more than would be expected by chance alone, and so this structure was not included in the best-fit model. Thus, in no case did the random guessing structure provide sufficient explanatory power to warrant inclusion in the final, best-fit model.

In summary, when considering how students processed MC and MTF questions, a reasonable initial model would have been one in which all students engaged in informed reasoning based on option attractiveness. A joint attractiveness value for each response option/statement could have been estimated and converted into a predicted selection/endorsement probability in the MC and MTF formats (Fig. 2). In practice, this model provides a poor approximation of observed student responses, particularly underestimating the proportion of students that selected the correct (A) and fully correct
(TFFF) answers in the MC and MTF formats, respectively. Additional structures helped account for the prevalence of certain observed response patterns. In the $\mathrm{MC}$ format, mastery and partial mastery each typically increased the proportion of students predicted to answer correctly. In the MTF format, mastery typically raised the predicted number of fully correct responses (TFFF), and partial mastery increased the predicted partially correct TTFF responses. Double-T endorsement bias slightly suppressed predicted TFFF responses while elevating all the response combinations with two true answers. The addition of these structures brought predicted responses into closer alignment with observed response rates, consistent with the notion that each structure represented an approximation of how subsets of students approached the questions.

In the MTF format, the selection of TFFF is most strongly influenced by mastery and is generally robust to the consequences of partial mastery and endorsement bias (Fig. 2). Partial mastery and endorsement bias can have fairly large effects on the rate of students that choose TTFF. Thus, quantifying partial mastery is a key asset of the MTF format that cannot be recapitulated by MC questions. Endorsement bias, while generally small, does cloud fine-scale interpretation of distractors.

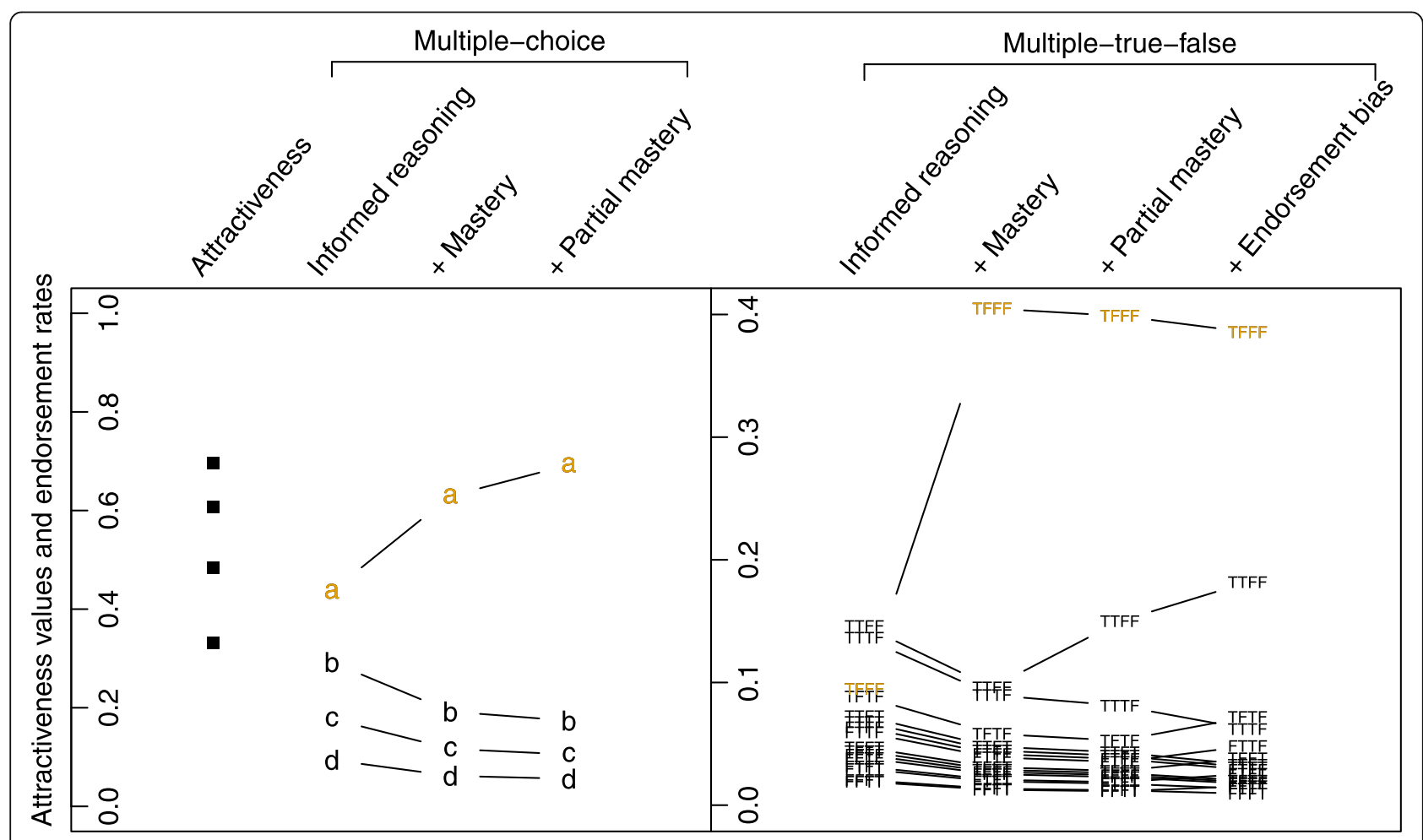

Fig. 2 Diagram illustrating how the addition of different components leads to changes in estimated parameter values. Panels show best-fit parameters averaged across all questions, with the right-hand panel re-scaled for clarity. For each format, the predicted proportion of student answers is first shown under a simplified model in which all students only use informed reasoning based on the attractiveness of the response options. Subsequent columns include the stated model component in addition to parameters to the left 
However, endorsement bias pulls students engaging in informed reasoning away from TFFF, distilling the selection of TFFF as a more honest representation of mastery.

\section{Format comparison}

Calculating parameter values for each structure provided an estimation of the proportion of students that operated according to a given structure for each question. Comparing observed student responses to these inferred values revealed the extent to which each format provided raw answers that adequately captured inferred student approaches. To understand the degree to which each format identified students with complete understandings of all the response options, we compared the rates at which students selected the correct response in the $\mathrm{MC}$ format or provided a fully correct answer in the MTF format to the inferred mastery level for each question (Fig. 3). We found that correct answer rates in the MC format overestimated inferred mastery to a large degree, and the degree of overestimation varied markedly among questions. Conversely, the fully correct response rate in the MTF format had close to a one-to-one relationship with inferred mastery levels.

The greater variation in $\mathrm{MC}$ questions could not be attributed to an unexplained error in the model (i.e., the inherent noise in choosing one out of four options), since predicted response rates closely matched observed response rates. A good fit is illustrated for both MC and MTF formats, without major bias. The greater differences between the open (predicted) and closed (observed) values for the multiple choice format is simply a consequence of the inherent noise in a four-option MC response as opposed to an effectively 16-option MTF response pattern. Conversely, the variance between $\mathrm{MC}$ correct responses and inferred mastery resulted from variance in the attractiveness of the response options comprising each question (i.e., some combinations of attractiveness values enabled the correct option to more closely approximate inferred mastery than other combinations). For example, distractors with low attractiveness would lead most students using informed reasoning to choose the correct answer in $\mathrm{MC}$, whereas relatively even attractiveness among distractors would result in students using informed reasoning to choose an incorrect answer in MC. In contrast, a fully correct MTF response provided a more consistent estimate of inferred mastery because MTF answer patterns were not as heavily influenced by the particular combination of statement attractiveness comprising each question.

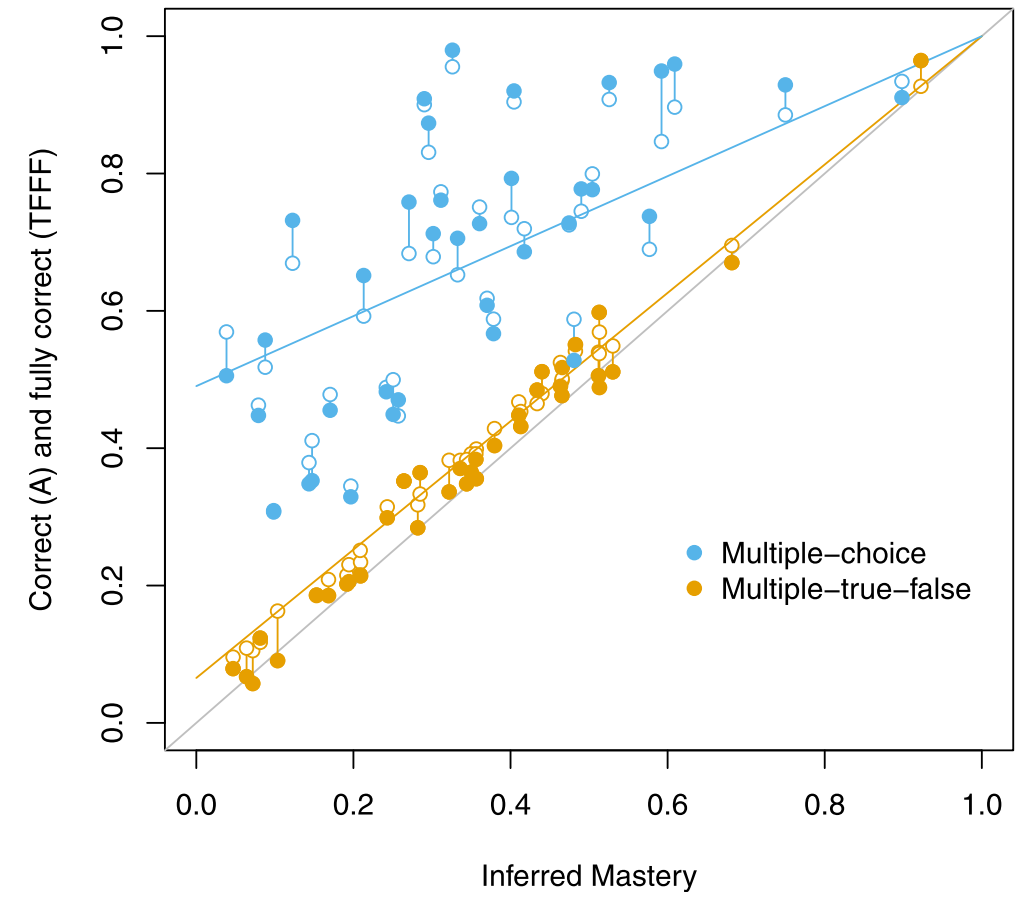

Fig. 3 Multiple-choice (MC) correct response rates provide a poor estimation of inferred mastery, while multiple-true-false (MTF) fully correct response rates provide a close approximation of inferred mastery. The $x$-axis represents the inferred mastery levels for each question derived from student responses in both formats. The $y$-axis represents the proportion of correct (A) or fully correct (TFFF) responses for MC and MTF questions, respectively. Closed circles represent observed values, and open circles represent predicted values. Vertical lines connect the observed and predicted values for an individual question in a particular format. The long solid lines represent the best fit of the parameter averages for each format. The one-to-one line is shown in solid gray 
To further understand how student answers corresponded to underlying question approaches, we visualized the proportion of students modeled as selecting each answer choice through a particular approach. In the MC format, some students selected the correct answer based on mastery, but many additional students also selected the correct answer based on partial mastery or informed reasoning (Fig. 4a). Thus, students could arrive at the correct answer through three distinct approaches. The mastery students chose the correct answer based on their correct understanding of all the options. The partial mastery students selected the correct $\mathrm{MC}$ answer, but they would have incorrectly endorsed the first distractor, if given the opportunity. The students using informed reasoning did not have certainty regarding any of the statements, but they chose the correct answer based on a comparison of the relative merits of each option. Finally, students could arrive at the incorrect answer through informed reasoning that involved comparing the attractiveness of the different answer options.

In the MTF format, student answers showed closer alignment with their approaches. Some students selected a fully correct answer based on mastery, while only a small proportion arrived at this answer pattern via informed reasoning (Fig. 4b). Some students selected a partially correct answer based on either partial mastery or informed reasoning. These answer patterns better reflected their proportional understanding of the various statements. Partial mastery students correctly answered three out of four statements, while students using informed reasoning earned credit in a manner that approximated their understanding of the four statements.

To contrast the differing information content in MC and MTF questions, we determined the extent to which responses from one format could predict response to the other format. Generally, the root-mean-square deviation (RMSD) of the data points from the one-to-one line was higher when $\mathrm{MC}$ data alone was used to estimate observed MTF statement endorsement rates (Fig. 5a), while MTF data provided a much closer estimation of observed MC option selection rates (Fig. 5b). Furthermore, for the comparison of MC statement a and MTF option a, which had similar RMSD, the use of MC data alone was biased (Piñeiro, Perelman, Guerschman, \& Paruelo, 2008) in the prediction of MTF endorsement, as indicated by a significantly non-zero intercept $(0.36, t=5.99$, $p<0.0001)$ and a slope significantly different from one $(-1.41, t=-5.23, p<0.0001)$. Conversely, the use of MTF data alone accurately predicted the MC selection

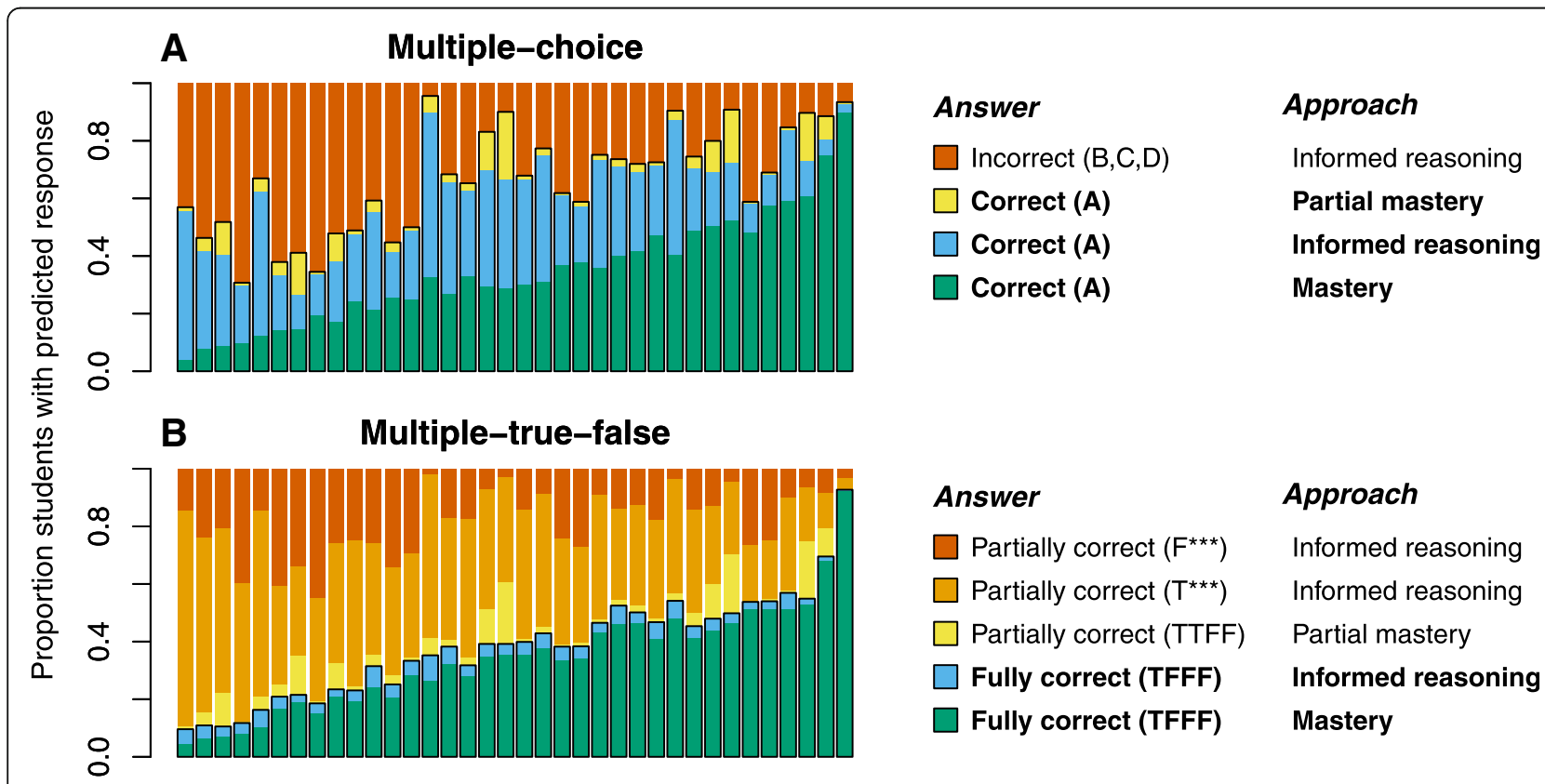

Questions in order of increasing inferred mastery

Fig. 4 Proportion of responses by category for each question in the a multiple-choice (MC) and $\mathbf{b}$ multiple-true-false (MTF) formats, as predicted by best-fit model parameters. Questions are ordered by average mastery level across the two formats, such that "harder" questions are to the left and "easier" questions are to the right. Bar colors represent the modeled proportion of students giving an answer pattern through a particular approach, as described in the legends. $T^{* * *}$ represents a correct answer to the true statement and at least one incorrect answer to one of the remaining false statements. $F^{* * *}$ represents an incorrect answer to the true statement and any combination of answers to the remaining statements. Correct and fully correct responses are bolded, which highlights the distribution of approaches by which students can select the correct MC answer as opposed to the MTF format where students achieve a fully correct answer more strictly through a mastery approach 


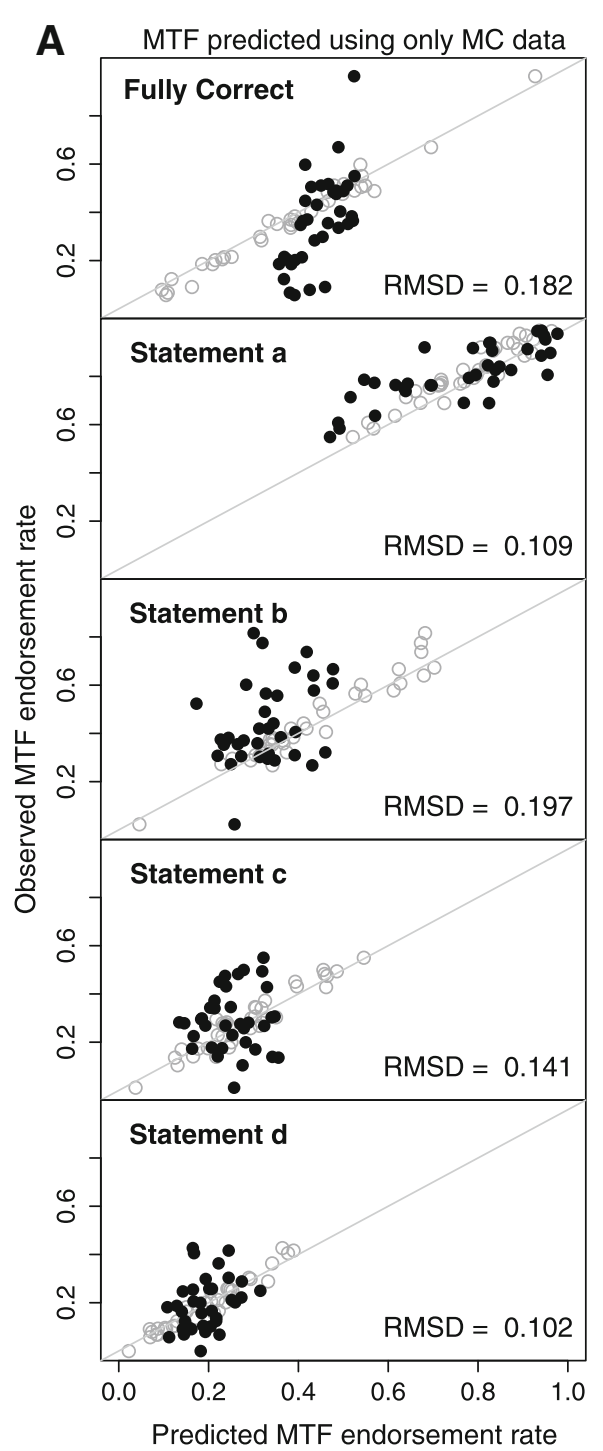

B MC predicted using only MTF data

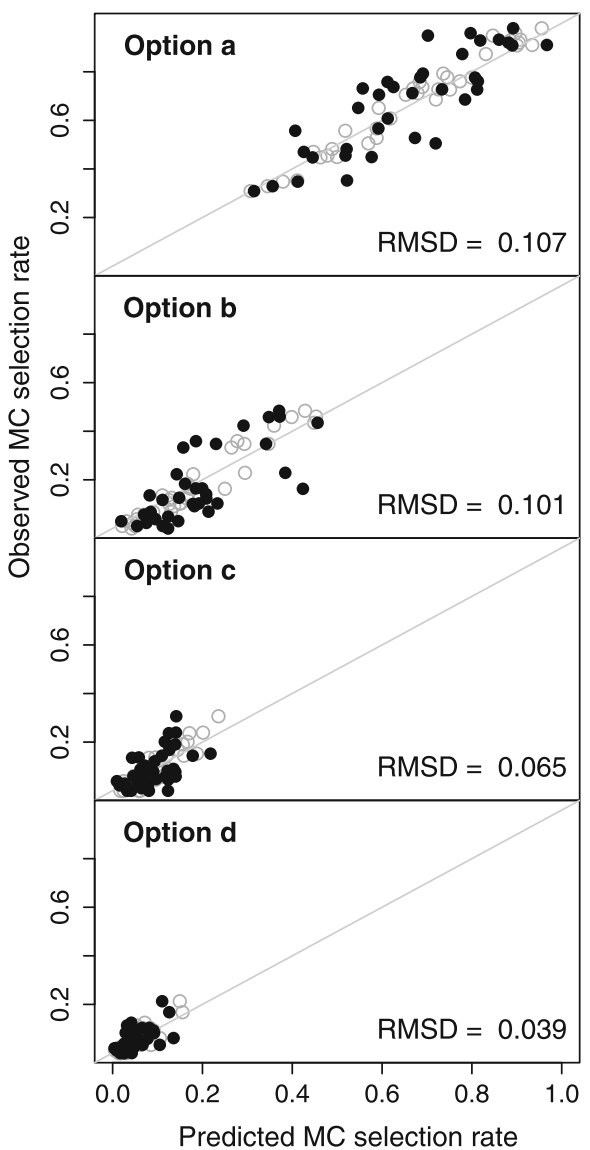

Fig. 5 a Multiple-choice (MC) data alone fails to predict the endorsement rates of multiple-true-false (MTF) statements, while $\mathbf{b}$ MTF data alone more closely predicts MC results. The black filled circles represent predicted values using only data from the $\mathbf{a}$ MC or $\mathbf{b}$ MTF format. For comparison purposes, the gray open circles represent predicted values using the full data set (i.e., including both MC and MTF response data). One-to-one lines are shown in solid gray. Root-mean-square deviation (RMSD) is the mean deviation from the one-to-one line in the units of endorsement or selection rate

rate, as indicated by an intercept that did not significantly differ from zero $(0.002, t=0.026, p=0.98)$ and a slope that did not significantly differ from one $(1.03, t=$ $0.253, p=0.80)$.

\section{Use of MTF format}

We also wished to understand theoretically how the relationship between MC correct responses and inferred mastery levels was affected by distractor characteristics. For students engaged in informed reasoning, the presence of more attractive distractors might draw students away from the correct answer and reveal their limited understandings. This is illustrated in two calculations in which the attractiveness values of the distractors were artificially changed (Fig. 6). In the first, all distractors were as attractive as the most attractive distractor (i.e., a practical limit), and in the second, the distractors were each as attractive as the correct option (i.e., a theoretical limit). While either of these conditions enabled MC correct responses to better approximate inferred mastery levels, the relationship still lay far from the one-to-one line. Conversely, the relationship for MTF questions lays close to the one-to-one line in all cases.

Finally, while our model provided estimations of the proportion of students that processed questions via different approaches, it would be impractical for an 


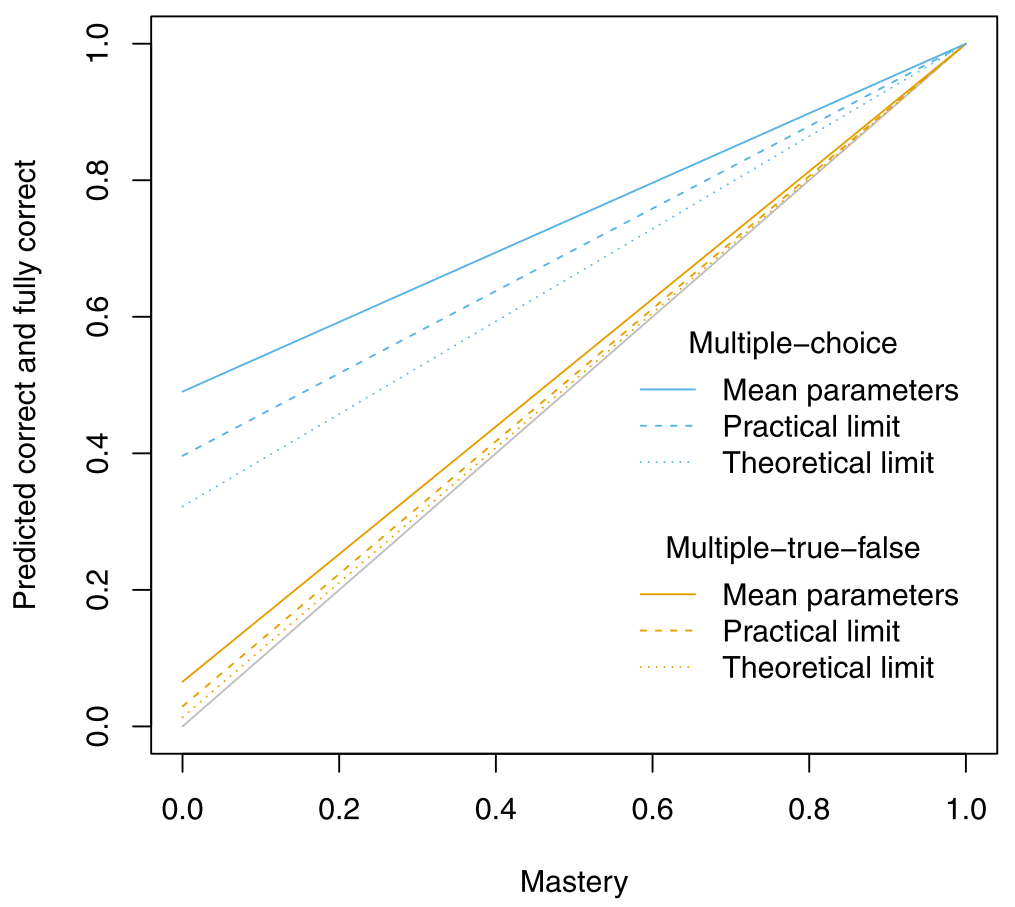

Fig. 6 How predicted responses relate to inferred mastery with different distractor characteristics. Blue and orange lines represent predicted correct/fully correct responses at the given inferred mastery level for multiple-choice (MC) and multiple-true-false (MTF) questions, respectively. Solid lines represent mean parameters from observed data, dashed lines represent the practical limit where all distractors are as attractive as the most attractive distractor, and dotted lines represent the theoretical limit where all distractors are as attractive as the correct answer. The one-toone line is shown in solid gray

instructor to fit a complex Bayesian model for each exam. Thus, we aimed to determine how instructors could estimate the extent to which students had fully achieved the specified learning objectives (i.e., mastery) and to diagnose where remaining students had residual difficulties (i.e., statement attractiveness). For MC questions, there was no way to use student responses to determine underlying mastery of all the question options because MC correct answer selection rates had an inconsistent relation to inferred mastery levels (see Fig. 3). Furthermore, MC selection rates for each answer option provided a relatively weak approximation of option attractiveness (Fig. 7a). For MTF questions, the proportion of students that gave a fully correct response (TFFF) correlated closely with inferred mastery levels (see Fig. 3). The MTF endorsement rates among students who did not answer fully correct also provided better approximations of the statement attractiveness values (Fig. 7a). In practice, endorsement bias had a very minimal effect on net endorsement rates per statement (Additional file 1: Supplementary Material 5).

Consequently, statement endorsement rates for MTF questions can be meaningfully processed by plotting the fraction of students correctly endorsing each statement, overlaid with a dashed line for the proportion of students answering fully correct (Fig. 7b). We have included processed results from four sample questions to illustrate how instructors can use MTF questions to understand question performance and prioritize feedback (Fig. 7b). In the first question, very few students provided a fully correct answer, indicative of low mastery levels. The students struggled with the false statements to varying degrees. For the second question, closer to half of the students provided a fully correct answer, while most of the remaining students incorrectly identified the first false statement as true. For the third and fourth question, a high proportion answered fully correct, but the remaining students struggled to identify the correct answer at various levels across the first three statements, including the true statement. In each case, the number of fully correct responses can serve as a close approximation of full student comprehension, while statement selection rates among the remaining students can help pinpoint areas where struggling students need additional support.

\section{Discussion}

How well do MC and MTF response rates approximate the proportion of students that have complete, partial, or little understanding of the response options?

With respect to ascertaining the degree of understanding, we found $\mathrm{MC}$ correct answers provided a poor 
A

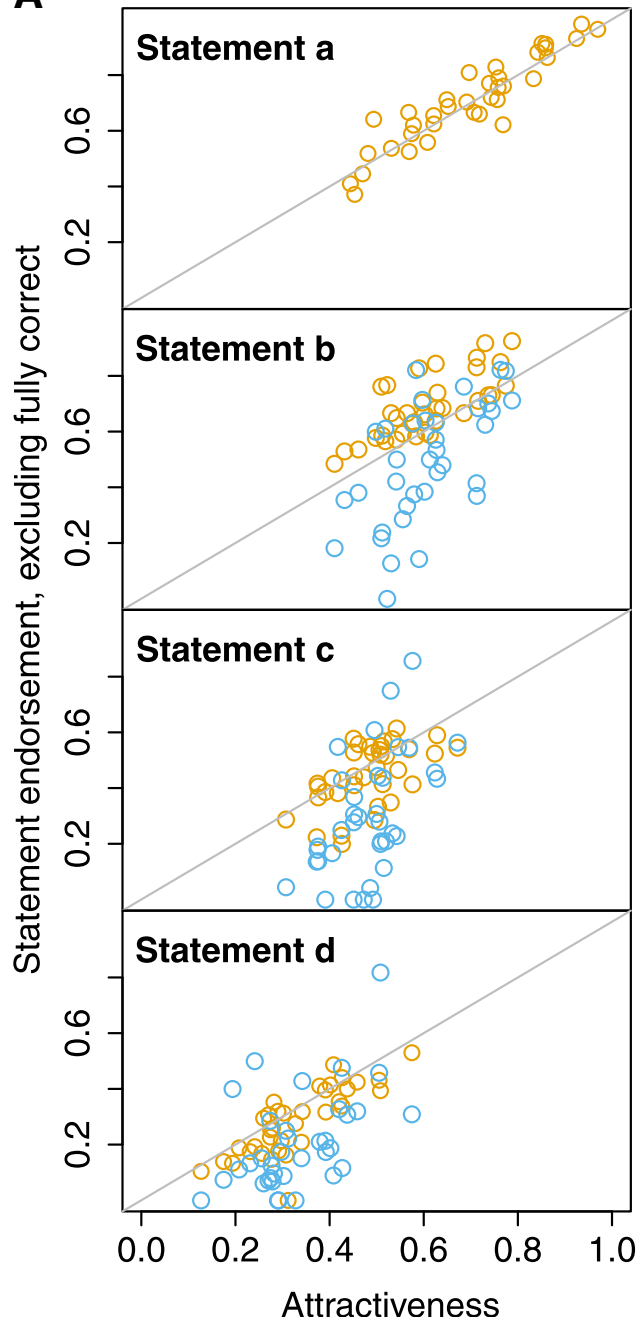

B

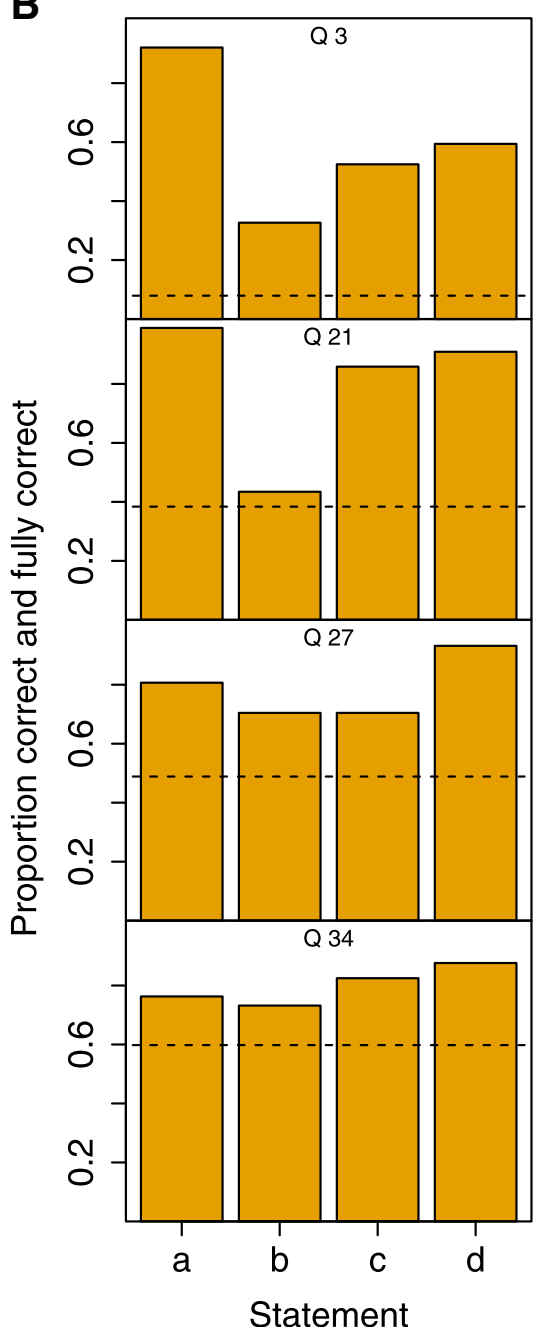

Fig. 7 a Relationship of multiple-choice (MC, blue circles) and multiple-true-false (MTF, orange circles) endorsement rates to option/statement attractiveness values across all questions among students not giving fully correct answers. $\mathbf{b}$ Response pattern from four representative MTF questions. The dashed line represents the proportion of students providing a fully correct answer (TFFF). The column labeled a represents the proportion of students that answered correctly the true statement, and the columns labeled b-d represent the proportion of students that answered correctly each false statement

estimation of a student's mastery of all the answer options. Indeed, the proportion of students answering a MC question correctly was substantially, but inconsistently, higher than the level of inferred question mastery (Fig. 3). As a consequence, the MC format failed to detect the large fraction of students who selected the correct answer based on partial mastery or informed reasoning of the various answer options (Fig. 4). Granted, while it is impossible to quantify partial mastery from MC responses alone, the finding that a partial mastery structure explains MTF responses demonstrates that MC questions inherently hide information on students inclined to simultaneously ascribe support to the correct answer and an incorrect answer.
Conversely, the proportion of students that gave a completely correct MTF answer (i.e., TFFF) correlated closely with inferred mastery (Fig. 3), and very few students achieved complete correctness based on informed reasoning (Fig. 4). In short, this is because of the information potential embedded in an MTF question. The combined MTF response across four statements represents one of 16 statement combinations, while an MC response can be only one of four outcomes. For students with incomplete understandings, $\mathrm{MC}$ selection rates provided poor estimations of the independent attractiveness of each option, whereas MTF statement responses correlated more closely with statement attractiveness (Fig. 7a). Taken together, these data provide compelling evidence that selection of the correct MC answer did not preclude 
misunderstanding of other options. Rather, the $\mathrm{MC}$ format masked important layers of student thinking that the MTF format revealed.

\section{To what extent do MC and MTF responses reflect random guessing?}

One of the most salient differences between the MC and MTF formats stems from the different theoretical rates at which students are able to guess the correct answer. Contrary to common expectations, we found that purely random guessing was not broadly characteristic of student responses. Instead, students with limited understandings were better modeled as making selections based on some type of informed reasoning. The informed reasoning structure has similarities to the "pseudo-guessing" parameter from IRT, which specifies the probability at which low performing students will select the correct answer (Hambleton, Swaminathan, \& Rogers, 1991). However, the informed reasoning structure has the additional benefit of accounting for mixed and partial understanding across options. This result also resonates with previous findings related to guessing behaviors on closed-ended questions. Indeed, Cronbach (1941) concluded decades ago that "No guess is a completely random response; even the student without knowledge consciously reacts to the tone and general character of the statement." Similarly, a meta-analysis of the MC literature found that students typically select the fourth and fifth options (ranked by student endorsement rates) less than $5 \%$ of the time and that removing these options has little effect on overall test reliability (Rodriguez, 2005). This meta-analysis underscores the notion that the theoretical guess rate (Fig. 3) has limited relevance for $\mathrm{MC}$ questions because students can normally eliminate certain options.

Given the potential for random guessing to artificially inflate student scores, assessment developers have devised approaches to either decrease guessing behaviors or apply post hoc guessing corrections (Chiu \& Camilli, 2013). For the MC format, test administrators often use formula scoring that discourages guessing by penalizing students for incorrect responses by an amount proportional to the number of answer options (Frary, 1988). This adjustment relies on the implicit assumption that a student who does not know the correct answer randomly chooses an option. Under this scoring scheme, students are advised to answer questions if they can eliminate at least one option and to leave questions blank if they have no knowledge of any of the options. By reducing the tendency to engage in random guessing, this scoring scheme has been shown to yield moderate increases in score reliabilities and validities (Alnabhan, 2002; Burton, 2002; Diamond \& Evans, 1973). However, this method also introduces confounding variables related to personality and risk-taking behaviors and fails to account for intermediate levels of understanding between fully correct and random guessing (Ávila \& Torrubia, 2004). Our results further call formula scoring into question on two levels. First, the finding that a random guessing term does not fit the model suggests that students rarely adopted this type of behavior in the given assessment context. Second, the finding that an appreciable number of students approach questions with either partial mastery or informed reasoning demonstrates that non-mastery students typically have some underlying knowledge or intuitions regarding a question.

\section{To what extent can responses for each format be used to predict responses to the other format?}

Correct MC responses were biased in predicting the proportion of students that endorsed the corresponding true statement in the MTF format. This was particularly manifest on challenging questions where observed MTF endorsement rates for true statements were higher than endorsement rates predicted by MC data alone (Fig. 5a, statement a). In other words, students were drawn away from selecting the correct option by a distractor in the MC format, but they were free to endorse the correct statement in the MTF format independent of the distractor.

Correct MC responses could not predict the rate of fully correct MTF responses, and the selection rates for MC distractors could not predict endorsement rates for false statements in the MTF format (Fig. 5a). MC questions were particularly poor at capturing the extent to which students believe incorrect ideas, likely because this format forces students to select only one answer when they may believe more than one to be correct. Conversely, the MTF format enables students to separately endorse each answer option, thus revealing student thinking in a manner that is less dependent on other options. As a consequence, MTF answer patterns alone fairly accurately predicted the selection rates for both the correct and incorrect $\mathrm{MC}$ answers (Fig. 5b). Thus, from an instructional standpoint, $\mathrm{MC}$ responses had more limited informational content than MTF responses, particularly with respect to the incorrect/false distractors.

What is the potential utility of MC, as compared to MTF, to reveal student question mastery, even with different distractor characteristics?

While we endeavored to write questions that could capture common correct and incorrect understandings related to a given conceptual area, we also wished to investigate theoretically how distractor characteristics influence the ability of questions to diagnose student conceptions. We reasoned that perhaps more attractive distractors would draw non-mastery students away in 
the $\mathrm{MC}$ format, thus revealing their lack of complete understanding. Our calculations not only illustrated how this could improve MC questions, but it also revealed the fundamental limitations of the $\mathrm{MC}$ format in comparison to the MTF format (Fig. 6). This difference between formats reflected the problem that, even with highly attractive distractors, a substantial number of students would have selected the MC correct answer based on partial mastery or informed reasoning. These findings support the notion that-for questions with independent response options-the $\mathrm{MC}$ format will have an intrinsic inability to estimate mastery of all the answer options because the options have been reduced to a forced, non-independent selection process.

\section{How can MTF responses be appropriately processed and interpreted by educators?}

We propose the following scheme for processing MTF responses for instructors. First, the percent of students giving a fully correct answer can be calculated for each question stem. These students provide a close estimation of the students who have achieved question mastery and reflect the relative extent to which the question remains problematic. Then, the percent correct for each $\mathrm{T} / \mathrm{F}$ statement can be calculated to determine where the remaining students are having difficulties. While this processing does not incorporate the statistical structure of the Bayesian model, it aligns closely with the statistical analysis while providing a relatively convenient way to parse assessment results according to two instructor goals. On one level, the instructor wants to know how many students have achieved mastery. On a second level, the instructor wants to know where students without mastery are still struggling in order to provide appropriate feedback.

\section{Conclusions}

When used for diagnostic purposes, the efficacy of closed-ended questions rests on the premise that selection of predefined response options can capture underlying student thinking (Adams \& Wieman, 2011). Building on our previous findings, we sought to address several research questions related to how MC and MTF questions reveal student understandings of the various response options. We chose to develop a variant of a Bayesian item response model because it enabled us to propose that certain types of student understanding exist within the population and simultaneously predict how these understandings become manifest in the MC and MTF formats. Our modeling took advantage of the crossover experimental design by fitting joint parameters for individual MC $\times$ MTF questions, which permitted us to test specific hypotheses regarding the relationship between question format-independent understandings and question format-dependent response tendencies. By comparing models with and without each model structure and alternative structures, we were able to determine the degree to which various latent student understandings and approaches were consistent with observed responses. Our analyses indicate that low performing students use informed reasoning based on option attractiveness but do not engage in uniform random guessing.

Our findings further support the notion that $\mathrm{MC}$ responses provide limited insight into the degree to which students believe the various answer options, while the MTF format provides a more detailed way to capture varying degrees of understanding within an individual question. This work provides an empirically grounded perspective to help address the stated need for theory on the relationship between item formats and cognitive behavior (Haladyna et al., 2002). In considering our results, it is important to note that our Bayesian analysis can only identify structures and parameters supported by the data. We must then interpret and align these with a given student processing approach. Given the inherent limitations of any forced-response format, there are additional layers of complexity that we cannot capture, such as the cognitive processes guiding informed reasoning (e.g., to what extent does statement attractiveness stem from underlying knowledge versus superficial item cues?). While the experimental questions compared in the Bayesian model were necessarily restricted to MTF questions with a single true statement, we have no reason to believe that this model of student processing would be substantially different for MTF questions with multiple true statements. We further note that the MTF format takes longer for a student to process than an equivalent MC question (Frisbie, 1992), but the moderate increase in time required is considerably outweighed by the several fold increase in response information provided.

Our assessment development process aligns with typical instructor practice but differs from the development of research-based assessments (RBAs) or concept inventories. We did not analyze overall student ability with respect to a broader construct, and our questions did not undergo student interviews or pilot testing. While it is possible that they would have different question properties, many of these MC-based instruments use questions where distractors are not mutually exclusive from the correct answer, and so we predict that they would similarly overestimate the degree to which students have question-level mastery. In light of this limitation, several recent RBAs have elected to use the MTF format (Couch et al., 2015, 2019; Semsar et al., 2019; Summers et al., 2018). In this context, the MTF format helps improve content validity through its ability to cover a broad 
range of concepts in a comparatively short time span as well as item generalizability by addressing a particular concept across multiple questions. Additional research is needed to determine the applicability of our findings to $\mathrm{MC}$ questions that have undergone a more thorough development process. Given the important role that research-based assessments have played in disciplinebased education research (National Research Council (NRC), 2012), understanding the properties of different question formats represents an important step to the proper interpretation and use of assessment results.

\section{Additional file}

Additional file 1: Supplementary Material 1. Student demographics and overall course performance. Supplementary Material 2.

Mathematical description of the most supported model. Supplementary Material 3. Calculating the probability of selecting each multiple-choice option. Supplementary Material 4. Best-fit distributions from which model parameters were drawn. Supplementary Material 5. Influence of endorsement bias on results interpretation. (DOCX $61 \mathrm{~kb}$ )

\section{Abbreviations}

AIC: Akaike information criterion; IRT: Item response theory; MC: Multiplechoice; MTF: Multiple-true-false; RMSD: Root-mean-square deviation: WAIC: Watanabe-Akaike information criterion

\section{Acknowledgements}

We thank the University of Nebraska-Lincoln (UNL) discipline-based education research (DBER) community for critical research discussions. We are grateful to our colleagues who provided feedback on project design and manuscript revisions: Kathleen Brazeal, Tanya Brown, Mary Durham, Joanna Hubbard, Michelle Howell, and Kelly Lane. This work was supported by an internal award from UNL.

\section{Funding}

This work was supported by an internal award from UNL.

\section{Availability of data and materials}

Source data will be uploaded to Dryad once the manuscript is published.

\section{Authors' contributions}

$B A C$ conducted data collection; CEB and BAC conceived of the analysis; CEB developed the model; CEB and BAC drafted and revised the manuscript. Both authors read and approved the final manuscript.

\section{Competing interests}

The authors declare that they have no competing interests.

\section{Publisher's Note}

Springer Nature remains neutral with regard to jurisdictional claims in published maps and institutional affiliations.

Received: 27 March 2018 Accepted: 10 April 2019

Published online: 10 May 2019

\section{References}

Adams, W. K., \& Wieman, C. E. (2011). Development and validation of instruments to measure learning of expert-like thinking. International Journal of Science Education, 33(9), 1289-1312. https://doi.org/10.1080/09500693.2010.512369.

Alnabhan, M. (2002). An empirical investigation of the effects of three methods of handling guessing and risk taking on the psychometric indices of a test. Social Behavior and Personality, 30, 645-652.

Angelo, T. A. (1998). Classroom assessment and research: An update on uses, approaches, and research findings. San Francisco: Jossey-Bass.
Ávila, C., \& Torrubia, R. (2004). Personality, expectations, and response strategies in multiple-choice question examinations in university students: A test of Gray's hypotheses. European Journal of Personality, 18(1), 45-59. https://doi. org/10.1002/per.506.

Baker, F. B., \& Kim, S.H. (2004). Item response theory: Parameter estimation techniques (2nd ed.). New York: Marcel Dekker.

Black, P., \& Wiliam, D. (2009). Developing the theory of formative assessment. Educational Assessment, Evaluation and Accountability, 21(1), 5-31. https://doi. org/10.1007/s11092-008-9068-5.

Bock, R. D. (1972). Estimating item parameters and latent ability when responses are scored in two or more nominal categories. Psychometrika, 37(1), 29-51. https://doi.org/10.1007/BF02291411.

Bolt, D. M., Cohen, A. S., \& Wollack, J. A. (2001). A mixture item response model for multiple-choice data. Journal of Educational and Behavioral Statistics, 26(4), 381-409.

Briggs, D., Alonzo, A., Schwab, C., \& Wilson, M. (2006). Diagnostic assessment with ordered multiple-choice items. Educational Assessment, 11(1), 33-63. https:// doi.org/10.1207/s15326977ea1101_2.

Burnham, K. P., \& Anderson, D. R. (2002). Model selection and multimodel inference: A practical information-theoretic approach (2nd ed.). New York: SpringerVerlag Retrieved from https://www.springer.com/us/book/9780387953649.

Burton, R. F. (2002). Misinformation, partial knowledge and guessing in true/false tests. Medical Education, 36(9), 805-811.

Chiu, T.-W., \& Camilli, G. (2013). Comment on 3PL IRT adjustment for guessing. Applied Psychological Measurement, 37(1), 76-86. https://doi.org/10.1177/ 0146621612459369

Couch, B. A., Hubbard, J. K., \& Brassil, C. E. (2018). Multiple-true-false questions reveal the limits of the multiple-choice format for detecting students with incomplete understandings. BioScience, 68(6), 455-463. https://doi.org/10. 1093/biosci/biy037.

Couch, B. A., Wood, W. B., \& Knight, J. K. (2015). The molecular biology capstone assessment: A concept assessment for upper-division molecular biology students. CBE-Life Sciences Education, 14(1), ar10. https://doi.org/10.1187/cbe. 14-04-0071.

Couch, B. A., Wright, C. D., Freeman, S., Knight, J. K., Semsar, K., Smith, M. K., et al. (2019). GenBio-MAPS: A programmatic assessment to measure student understanding of vision and change core concepts across general biology programs. CBE-Life Sciences Education, 18(1), ar1. https://doi.org/10.1187/ cbe.18-07-0117.

Cronbach, L. J. (1941). An experimental comparison of the multiple true-false and multiple multiple-choice tests. Journal of Educational Psychology, 32(7), 533.

Crouch, C. H., \& Mazur, E. (2001). Peer instruction: Ten years of experience and results. American Journal of Physics, 69(9), 970-977. https://doi.org/10.1119/1. 1374249.

de Ayala, R. J. (2008). The theory and practice of item response theory (1st ed.). New York: The Guilford Press.

Diamond, J., \& Evans, W. (1973). The correction for guessing. Review of Educational Research, 43(2), 181-191.

Dudley, A. (2006). Multiple dichotomous-scored items in second language testing: Investigating the multiple true-false item type under normreferenced conditions. Language Testing, 23(2), 198-228. https://doi.org/10. 1191/0265532206lt327oa.

Eagan, K., Stolzenberg, E. B., Lozano, J. B., Aragon, M. C., Suchard, M. R., \& Hurtado, S. (2014). Undergraduate teaching faculty: The 2013-2014 HERI faculty survey. Los Angeles: Higher Education Research Institute, UCLA Retrieved from https:// www.heri.ucla.edu/monographs/HERI-FAC2014-monograph-expanded.pdf.

Ellis, A. P. J., \& Ryan, A. M. (2003). Race and cognitive-ability test performance: The mediating effects of test preparation, test-taking strategy use and selfefficacy. Journal of Applied Social Psychology, 33(12), 2607-2629. https://doi. org/10.1111/j.1559-1816.2003.tb02783.x.

Ericsson, K. A., Krampe, R. T., \& Tesch-romer, C. (1993). The role of deliberate practice in the acquisition of expert performance. Psychological Review, 100(3), 363-406.

Fox, J. (2010). Bayesian item response modeling. New York: Springer.

Frary, R. B. (1988). Formula scoring of multiple-choice tests (correction for guessing). Educational Measurement: Issues and Practice, 7(2), 33-38. https:// doi.org/10.1111/j.1745-3992.1988.tb00434.x.

Frey, B. B., Petersen, S., Edwards, L. M., Pedrotti, J. T., \& Peyton, V. (2005). Itemwriting rules: Collective wisdom. Teaching and Teacher Education: An International Journal of Research and Studies, 21(4), 357-364.

Frisbie, D. A. (1992). The multiple true-false item format: A status review. Educational Measurement: Issues and Practice, 11(4), 21-26. 
Frisbie, D. A., \& Sweeney, D. C. (1982). The relative merits of multiple true-false achievement tests. Journal of Educational Measurement, 19(1), 29-35. https:// doi.org/10.1111/j.1745-3984.1982.tb00112.x.

Gelman, A. (2006). Prior distributions for variance parameters in hierarchical models (comment on article by Browne and Draper). Bayesian Analysis, 1(3), 515-534.

Gelman, A., Hwang, J., \& Vehtari, A. (2014). Understanding predictive information criteria for Bayesian models. Statistics and Computing, 24(6), 997-1016. https://doi.org/10.1007/s11222-013-9416-2.

Haladyna, T. M., Downing, S. M., \& Rodriguez, M. C. (2002). A review of multiplechoice item-writing guidelines for classroom assessment. Applied Measurement in Education, 15(3), 309-333. https://doi.org/10.1207/S15324818AME1503_5.

Hambleton, R. K., Swaminathan, H., \& Rogers, H. J. (1991). Fundamentals of item response theory. Newbury Park: SAGE Publications, Inc.

Handelsman, J., Miller, S., \& Pfund, C. (2007). Scientific teaching. New York: W. H. Freeman and Co.

Hestenes, D., Wells, M., \& Swackhamer, G. (1992). Force concept inventory. The Physics Teacher, 30(3), 141-158.

Hubbard, J. K., \& Couch, B. A. (2018). The positive effect of in-class clicker questions on later exams depends on initial student performance level but not question format. Computers \& Education, 120, 1-12. https://doi.org/10. 1016/j.compedu.2018.01.008

Javid, L. (2014). The comparison between multiple-choice (mc) and multiple truefalse (mtf) test formats in Iranian intermediate EFL learners' vocabulary learning. Procedia - Social and Behavioral Sciences, 98, 784-788. https://doi. org/10.1016/j.sbspro.2014.03.482.

Kalas, P., O'Neill, A., Pollock, C., \& Birol, G. (2013). Development of a meiosis concept inventory. CBE-Life Sciences Education, 12(4), 655-664. https://doi.org/ 10.1187/cbe.12-10-0174

Kim (Yoon), Y. H., \& Goetz, E. T. (1993). Strategic processing of test questions: The test marking responses of college students. Learning and Individual Differences, 5(3), 211-218. https://doi.org/10.1016/1041-6080(93)90003-B.

Kreiter, C. D., \& Frisbie, D. A. (1989). Effectiveness of multiple true-false items. Applied Measurement in Education, 2(3), 207-216.

National Research Council (NRC). (2012). Discipline-based education research: Understanding and improving learning in undergraduate science and engineering. Washington, D.C.: National Academies Press.

Nehm, R. H., \& Reilly, L. (2007). Biology majors' knowledge and misconceptions of natural selection. BioScience, 57(3), 263-272. https://doi.org/10.1641/B570311.

Nehm, R. H., \& Schonfeld, I. S. (2008). Measuring knowledge of natural selection: A comparison of the CINS, an open-response instrument, and an oral interview. Journal of Research in Science Teaching, 45(10), 1131-1160. https:// doi.org/10.1002/tea.20251.

Newman, D. L., Snyder, C. W., Fisk, J. N., \& Wright, L. K. (2016). Development of the Central Dogma Concept Inventory (CDCl) assessment tool. CBE-Life Sciences Education, 15(2), ar9. https://doi.org/10.1187/cbe.15-06-0124.

Parker, J. M., Anderson, C. W., Heidemann, M., Merrill, J., Merritt, B., Richmond, G., \& Urban-Lurain, M. (2012). Exploring undergraduates' understanding of photosynthesis using diagnostic question clusters. CBE-Life Sciences Education, 11(1), 47-57. https://doi.org/10.1187/cbe.11-07-0054.

Piñeiro, G., Perelman, S., Guerschman, J. P., \& Paruelo, J. M. (2008). How to evaluate models: Observed vs. predicted or predicted vs. observed? Ecological Modelling, 216(3), 316-322. https://doi.org/10.1016/j.ecolmodel. 2008.05.006.

Pomplun, M., \& Omar, H. (1997). Multiple-mark items: An alternative objective item format? Educational and Psychological Measurement, 57(6), 949-962.

Rasch, G. (1960). Probabilistic models for some intelligence and attainments tests. Copenhagen: Danish Institute for Educational Research.

Rodriguez, M. C. (2005). Three options are optimal for multiple-choice items: A meta-analysis of 80 years of research. Educational Measurement: Issues and Practice, 24(2), 3-13. https://doi.org/10.1111/j.1745-3992.2005.00006.X.

Semsar, K., Brownell, S., Couch, B. A., Crowe, A. J., Smith, M. K., Summers, M. M. et al. (2019). Phys-MAPS: A programmatic physiology assessment for introductory and advanced undergraduates. Advances in Physiology Education, 43(1), 15-27. https://doi.org/10.1152/advan.00128.2018.

Smith, M. K., Wood, W. B., \& Knight, J. K. (2008). The Genetics Concept Assessment: A new concept inventory for gauging student understanding of genetics. CBE-Life Sciences Education, 7(4), 422-430. https://doi.org/10.1187/ cbe.08-08-0045

Stan Development Team. (2017). Stan modeling language users guide and reference manual, version 2.15 .0 (version 2.15.0). http://mc-stan.org.
Stenlund, T., Eklöf, H., \& Lyrén, P.-E. (2017). Group differences in test-taking behaviour: An example from a high-stakes testing program. Assessment in Education: Principles, Policy \& Practice, 24(1), 4-20. https://doi.org/10.1080/ 0969594X.2016.1142935.

Summers, M. M., Couch, B. A., Knight, J. K., Brownell, S. E., Crowe, A. J., Semsar, K., et al. (2018). EcoEvo-MAPS: An ecology and evolution assessment for introductory through advanced undergraduates. CBE-Life Sciences Education, 17(2), ar18. https://doi.org/10.1187/cbe.17-02-0037.

Thissen, D., Steinberg, L., \& Fitzpatrick, A. R. (1989). Multiple-choice models: The distractors are also part of the item. Journal of Educational Measurement, 26(2), 161-176. https://doi.org/10.1111/j.1745-3984.1989.tb00326.x.

Vehtari, A., Gelman, A., \& Gabry, J. (2017). Practical Bayesian model evaluation using leave-one-out cross-validation and WAIC. Statistics and Computing, 27(5), 1413-1432. https://doi.org/10.1007/s11222-016-9696-4.

Vickrey, T., Rosploch, K., Rahmanian, R., Pilarz, M., \& Stains, M. (2015). Researchbased implementation of peer instruction: A literature review. CBE-Life Sciences Education, 14(1), es3. https://doi.org/10.1187/cbe.14-11-0198.

Wood, W. (2004). Clickers: A teaching gimmick that works. Developmental Cell, 7(6), 796-798. https://doi.org/10.1016/j.devcel.2004.11.004.

\section{Submit your manuscript to a SpringerOpen ${ }^{\circ}$ journal and benefit from:}

- Convenient online submission

Rigorous peer review

- Open access: articles freely available online

- High visibility within the field

- Retaining the copyright to your article

Submit your next manuscript at $>$ springeropen.com 